\title{
The HCN4 Channel Mutation D553N Associated With Bradycardia Has a C-linker Mediated Gating Defect
}

\author{
Michael F. Netter ${ }^{\mathrm{a}}$ Marylou Zuzarte ${ }^{\mathrm{b}}$ Günter Schlichthörl ${ }^{\mathrm{b}}$ Nikolaj Klöcker ${ }^{c}$ Niels Decher
}

\begin{abstract}
aInstitute of Physiology and Pathophysiology, Vegetative Physiology Group, Philipps University of Marburg; 'DInstitute of Physiology and Pathophysiology, Cardiovascular Cellphysiology Group, Philipps University of Marburg; Institute of Neuro- and Sensory Physiology, Medical Faculty, University of Düsseldorf
\end{abstract}

\section{Key Words}

$\mathrm{D} 553 \mathrm{~N} \cdot \mathrm{cAMP} \cdot \mathrm{HCN} \cdot$ Cyclic nucleotide-binding domain $・$ C-linker $・$ Arrhythmia

\begin{abstract}
Background/Aims: The D553N mutation located in the C-linker of the cardiac pacemaker channel HCN4 is thought to cause sino-atrial dysfunction via a pronounced dominantnegative trafficking defect. Since HCN4 mutations usually have a minor defect in channel gating, it was our aim to further characterize the disease causing mechanism of D553N. Methods: Fluorescence microscopy, FACS, TEVC and patch-clamp recordings were performed to characterize D553N. Results: Surprisingly, we found that D553N channels reach the plasma membrane and have no apparent trafficking defect. Co-expression of D553N with HCN4 also revealed no dominant-negative effect on wild-type channels. Consistent with the normal cell surface expression of D553N, it was possible to extensively characterize D553N mutants in Xenopus oocytes and mammalian cells. D553N channels generate currents with reduced amplitude, while the kinetics of activation and deactivation are not altered. While the regulation of $\mathrm{D} 553 \mathrm{~N}$ by tyrosine kinases is normal, we observed a change in the cAMP regulation which however cannot account for the strong loss-of-function of the mutant. Conclusion: The pronounced current reduction and the regular surface expression indicate a major gating defect of the C-linker gate. We hypothesize that the D553N mutation stabilizes a previously reported salt bridge important for the gating of the channel.
\end{abstract}




\section{Introduction}

Mutations in genes encoding cardiac ion channels or their subunits cause a variety of arrhythmias, including long QT syndrome, short QT syndrome, Brugada syndrome and conduction disorders [1]. While some of the inherited forms of arrhythmias, like the LQT syndrome, are quite well understood, little is known about gene defects involved in inherited forms of bradycardia and sinus node dysfunction such as the sick sinus syndrome. Patients with sick sinus syndrome suffer from bradycardia, sino-atrial block or sinus arrest, with episodes of atrial tachycardia. Mutations in the genes of the sodium channel SCN5A [2] and the pacemaker channel HCN4 have been reported to cause sick sinus syndrome [3]. Ion channels of the HCN family are hyperpolarization activated cation channels modulated by intracellular cAMP [4], phosphoinositides [5] and tyrosine phosphorylation [6]. Binding of cAMP to the cyclic nucleotide-binding domain (CNBD) of HCN channels leads to a positive shift in the voltage dependence of activation, resulting in an apparent speeding of activation [4].

In order to clarify the contribution of HCN channels to cardiac pacemaking, HCN knock-out mice were extensively studied [7-9]. Global and cardiac specific knock-out of the HCN4 gene leads to embryonic lethality between day 9.5 and 11.5 [8]. Although heart rate in isolated $\mathrm{HCN} 4 \%$ embryonic hearts is reduced by $40 \%$ and the chronotropic response to elevated cAMP levels is lost, no arrhythmias were detectable and hearts contracted regularly. Strikingly, there is no pacemaker current measurable from isolated embryonic $\mathrm{HCN}^{-/}$cardiomyocytes which indicates the importance of the HCN4 isoform for normal heart function [8]. In contrast, global HCN2 knock-out mice are viable, but suffer from a neuronal and cardiac phenotype [9]. A loss of HCN2 leads to hyperpolarization of the cell with the result of delayed action potentials leading to sinus arrhythmia characterized by varying RR-intervals [9]. However, so far no HCN2 mutations were identified in patients with sino-atrial arrhythmias.

Four different C-terminal HCN4 channel mutations have been described to cause sinoatrial arrhythmias [3, 10-12]. The HCN4 mutation 573X [3] has a premature stop codon in the C-terminus leading to a complete loss of the CNBD. Despite this truncation, the 573X mutant channels generate pacemaker currents. However, the patients suffer from bradycardia and chronotropic insufficiency, since the mutant channels lack response to cAMP. The mutation $695 X$ [12] lacks parts of the CNBD. Insertion of 13 nucleotides in the region coding for the CNBD leads to a frame shift resulting in a premature stop codon. Similar as for the 573X mutation, patients with the $695 \mathrm{X}$ mutation suffer from bradycardia and the mutants are resistant to cAMP modulation. The HCN4 mutation S672R [11] is located in the CNBD. Bradycardia in patients with an S672R exchange in the HCN4 channels is caused by a negative shift in the voltage dependence of channel activation, while the modulation by cAMP is unchanged.

According to these authors, the three disease causing HCN4 mutations in the C-terminus (573X, 695X and S672R) only show a mild loss-of-function. In contrast, the mutation D553N leads to an almost complete loss of channel function. D553N HCN4 channels were reported to have a trafficking defect which acts on co-expressed wild-type channels in a dominantnegative manner [10]. Interestingly, in contrast to other C-terminal HCN4 channel mutations, the D553N exchange is not located in the CNBD, but in the C-linker of the HCN4 channel. The C-linker, connecting the CNBD with the S6 segment and the channel pore, is known to mediate cAMP dependent changes in HCN channel gating [11]. The C-linker contributes to the cytosolic "gating ring" [13] of HCNs. In addition, it regulates channel gating by forming salt bridges with the S4-S5 linker [14] or the neighbouring subunit and the CNBD [15-16].

While sino-atrial arrhythmias by HCN4 mutations are often caused by minor loss-offunction in channel gating, D553N was reported to have a strong and dominant-negative trafficking defect $[10,17]$. Our aim was to characterize the disease causing mechanism of D553N HCN4 channels in more detail. Surprisingly, we found that D553N mutant channels 
do not have a dominant-negative trafficking defect and conduct currents with normal kinetics. The pronounced current reduction and the regular surface expression indicate a major gating defect of the $\mathrm{C}$-linker gate. Our study is in line with previous reports $[11,13-14]$ highlighting the role of the $\mathrm{C}$-linker in $\mathrm{HCN}$ channel gating.

\section{Materials and Methods}

\section{Ethical approval}

The investigation conforms to the guide for the Care and Use of laboratory Animals (NIH Publ. 85-23).

Molecular biology

QuikChange Site-Directed Mutagenesis Kit (Stratagene) was used to introduce mutations into human HCN4 cDNA. The constructs for oocyte expression were subcloned into pBF1 vector and cRNA was prepared with mMessage mMachine SP6 Kit (Ambion) after linearization with AdeI (Fermentas).

\section{Expression of HCN channels in Xenopus oocytes}

Xenopus oocytes were prepared as previously described [14]. Briefly, ovarian lobes were dissected from mature Xenopus laevis anaesthetized with tricaine. Subsequently, the lobes were digested with collagenase ( $1 \mathrm{mg} / \mathrm{ml}$, Worthington, type II) in OR2 solution containing (in mM): $\mathrm{NaCl} 82.5, \mathrm{KCl} 2, \mathrm{MgCl}_{2} 1, \mathrm{HEPES} 5$, (pH 7.4) for 120 minutes. Isolated oocytes were stored at $18{ }^{\circ} \mathrm{C}$ in ND96 recording solution containing (in $\mathrm{mM}$ ): $\mathrm{NaCl} 96, \mathrm{KCl} 2, \mathrm{CaCl}_{2} 1.8, \mathrm{MgCl}_{2} 1$, $\mathrm{HEPES} 5$, (pH 7.4) with added Na-pyruvate (275 mg/l), theophylline $(90 \mathrm{mg} / \mathrm{l})$ and gentamicin $(50 \mathrm{mg} / \mathrm{l})$. Stage IV and V oocytes were injected with $6.5 \mathrm{ng}$ of HCN4 cRNA. Standard two-electrode voltage-clamp experiments were performed 2-4 days after cRNA injection at room temperature $\left(21-22^{\circ} \mathrm{C}\right)$ in ND66 recording solution containing (in mM): $\mathrm{NaCl} 66, \mathrm{KCl} 32, \mathrm{CaCl}_{2} 1.8, \mathrm{MgCl}_{2} 1$, HEPES 5, (pH 7.4). Microelectrodes were fabricated from glass pipettes filled with $3 \mathrm{M} \mathrm{KCl}$. Tip resistance was in the range of 0.5-1.0 M $\Omega$. TEVC recordings were performed using a TurboTEC-10CD Amplifier (npi) with a Digidata 1200 A/D-converter (Axon Instruments). For data acquisition the software pCLAMP7 (Axon Instruments) was used and data were analyzed with ClampFit10 (Molecular Devices).

\section{Patch-clamp of HCN4 transfected COS-7 cells}

Cells were grown on $35 \mathrm{~mm}$ dishes (NUNC) to a confluency of about $50 \%$. Cells in each dish were transfected with $1 \mu \mathrm{g}$ of pEGFP-C1-HCN4 cDNA using Fugene 6 (Roche). After 24-48 hours, COS-7 cells were recorded in the whole cell configuration at room temperature $\left(21-22^{\circ} \mathrm{C}\right)$. Pipettes had a tip resistance of 2.5-4.0 M $\Omega$ when filled with a solution containing (in mM): $\mathrm{KCl} 130, \mathrm{NaCl} 10, \mathrm{MgCl}_{2} 0.5$, EGTA 1, and HEPES 5, (pH 7.4). Cells were bathed in a solution containing (in mM): $\mathrm{NaCl} 110, \mathrm{KCl} 30, \mathrm{MgCl}_{2} 0.5, \mathrm{CaCl}_{2} 1.8$ and HEPES 5, (pH 7.4). Series resistances were automatically compensated by $70 \%$.

\section{Drugs}

Stocks of genistein, daidzeine and isobutyl-methyl-xanthine (IBMX) were stored in DMSO and the final DMSO concentration in the bath solution did not exceed $0.1 \%$. All drugs used were obtained from SigmaAldrich.

\section{Fluorescence imaging}

COS-7, HeLa or HL-1 cells were grown on $35 \mathrm{~mm}$ glass bottom Petri dishes (WillCo) to a confluency of about $50 \%$. COS-7 and HeLa cells were grown in DMEM medium containing $10 \%$ fetal bovine serum (Invitrogen) and HL-1 cells were grown in Claycomb medium as previously described [18]. For the detection of HCN4 channels at the plasma membrane we introduced an extracellular HA-epitope in the S3-S4 linker, following residue R362. After 24 hours, cells were transfected with either $1 \mu \mathrm{g}$ pEGFP-C1-HCN4 or pEGFPC1-HCN4-HA per dish using Lipofectamine 2000 (Invitrogen). Cells were maintained at $37{ }^{\circ} \mathrm{C}$ for $6-48$ hours aerated with $5 \% \mathrm{CO}_{2}$. For imaging of fixed cells, HeLa cells were fixed with $4 \%$ paraformaldehyde in PBS, washed and labelled with monoclonal anti-HA high affinity antibody (Clone 3F10, Roche). Goat antirat IgG, F(ab') $)_{2}$ Texas Red (Jackson ImmunoResearch Laboratories Inc.) was used as secondary antibody. 
Fluorescence microscopy was performed with an Olympus IX71 microscope equipped with a $60 \times$ N.A. 1.3 PL APO objective or a 100× N.A. 1.4 PL APO objective (Olympus), standard EGFP/Texas Red filter sets and a cooled 12-bit CCD camera (SensiCam QE). During live cell imaging, cells were maintained at $37{ }^{\circ} \mathrm{C}$ using an objective heater (Bioptechs). Digital images were processed using Image-Pro Plus 6.0 (MediaCybernetics).

\section{FACS experiments}

Cells were grown in $60 \mathrm{~mm}$ petri dishes (NUNC) and transfected after 24 hours with $4 \mu \mathrm{g}$ pEGFPC1-HCN4-HA wild-type or a D553N mutant channel using Lipofectamine 2000. Cells were fixed 10 hours after transfection with $4 \%$ paraformaldehyde, washed and labeled using monoclonal anti-HA high affinity antibody (Clone 3F10) as primary and goat anti-rat IgG F(ab') -PE-Cy5 (Santa Cruz Biotechnology) as secondary antibody. Cells were then analyzed on a Calibur FACS system (BD Biosciences). EGFP and PE-Cy5 were excited with a $488 \mathrm{~nm}$ laser using FL1 channel and 505LP filter or using the FL3 channel and a 670LP filter, respectively. After adjusting for compensation and detection of EGFP positive cells without secondary antibody, the regions of interest were set accordingly.

\section{Data analysis}

Results are reported as mean \pm S.E.M. ( $\mathrm{n}=$ number of cells). Statistical differences were evaluated using a Student's t-test. Significance was assumed for $\mathrm{p}<0.05$, indicated by an asterisk (*). "n.s." indicates a non-significant change.

\section{Results}

Fluorescence imaging shows that HCN4 D553N mutant channels traffic to the plasma membrane

The disease causing HCN4 channel mutant D553N was reported to have a very pronounced trafficking defect when expressed in COS-7 cells [10]. In order to further characterize the disease causing mechanism of this HCN4 mutant, we performed fluorescence imaging experiments. Therefore, wild-type HCN4 channels (EGFP-HCN4) and HCN4-D553N mutant channels (EGFP-D553N) were labelled with an N-terminal EGFP tag. We used COS-7 cells, since those were also used for the initial description of D553N [10]. Transient transfection of COS-7 cells with EGFP-HCN4 led to fluorescence at the plasma membrane and in a perinuclear compartment (Fig. 1A) which is consistent with previously described data [19]. Surprisingly, EGFP-D553N showed a similar expression pattern as wild-type channels (Fig. 1A, B), preferentially residing in the same cellular compartments. We observed membrane expression of D553N mutant channels within 12 to 24 hours after transfection.

To gain further evidence that the D553N-mutant channels have a normal trafficking, we analyzed wild-type and D553N expression patterns in two additional cell lines. First, EGFPHCN4 and EGFP-D553N were detected at the plasma membrane of HeLa cells (Fig. 1C, D) which are more suitable to display plasma membrane expression. Next, we tested surface expression of D553N channels in the cardiac cell line HL-1 [18], to assess trafficking in a more physiological cell type for HCN channels. In this setting, D553N channels were also localized at the plasma membrane to a similar extent as wild-type channels (Fig. 1E, F). The latter experiments indicate that D553N channels might also reach the plasma membrane in cardiac tissue. Thus, in contrast to the initial report [10], D553N mutants reach the plasma membrane and have a similar cellular distribution pattern as wild-type HCN4 channels.

FACS analysis revealed no significant difference in surface expression of wild-type and D553N channels

To quantify whether wild-type and D553N HCN4 channels reach the plasma membrane with a similar efficiency, surface expression was quantified utilizing a fluorescence activated cell sorter (FACS). For these experiments, an extracellular HA-tag was introduced into the EGFP-HCN4 (EGFP-HCN4-HA) and EGFP-D553N (EGFP-D553N-HA) construct, respectively. EGFP-HCN4-HA transfected HeLa cells were fixed 12 hours after transfection and analyzed 

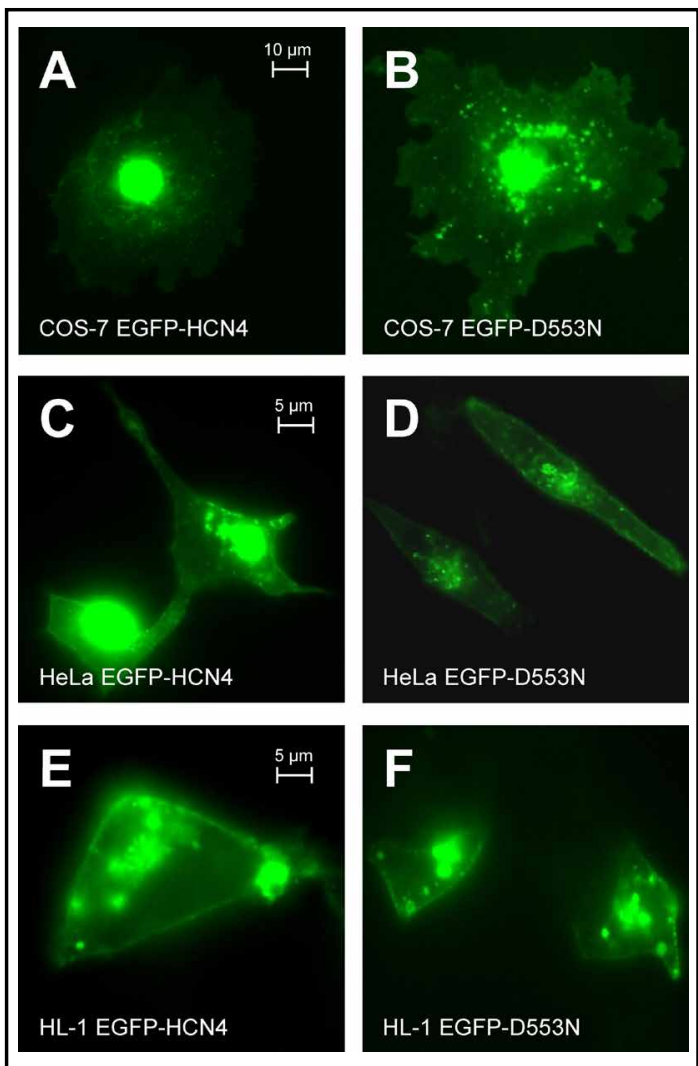

Fig. 1. Live cell imaging of HCN4 and D553N in different cell types. COS-7 cells transiently transfected with (A), EGFP-HCN4 and (B), EGFP-D553N. (C-D), HeLa cells transiently transfected with EGFP-HCN4 or EGFP-D553N. (E-F), EGFP-HCN4 or EGFP-D553N were transfected into the cardiac cell line HL-1. (A-F) All images were taken after $12 \mathrm{~h}$.

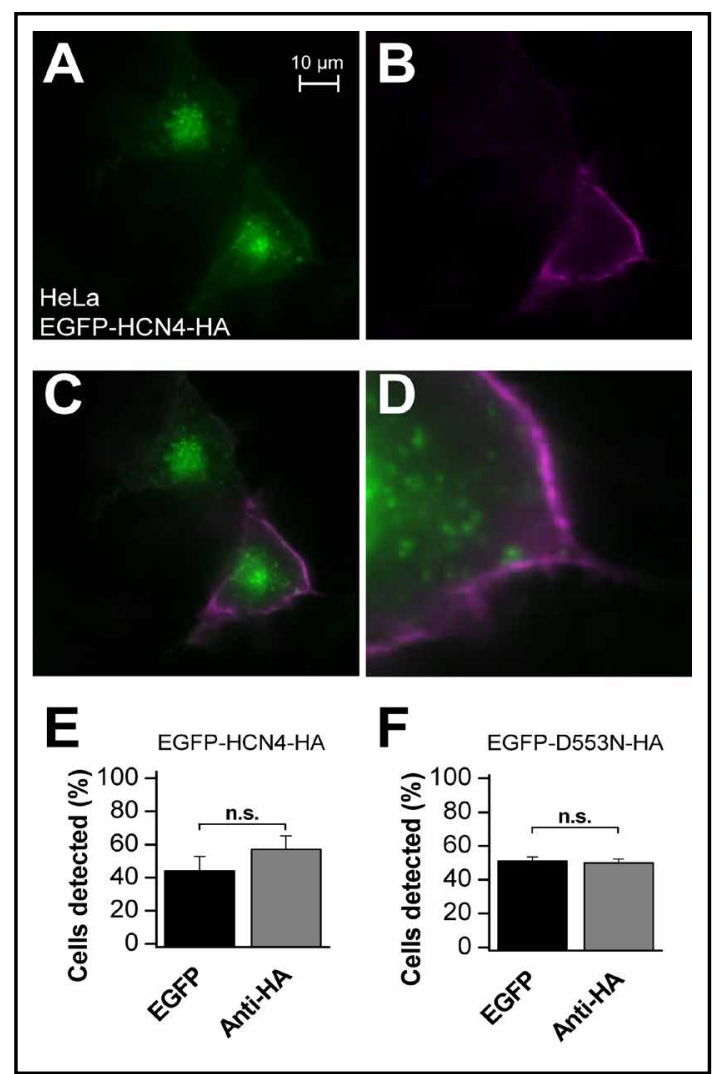

Fig. 2. Quantification of surface expression of HCN4 and D553N using FACS. (A), HeLa cells were transiently transfected with EGFP-HCN4-HA and fixed after $12 \mathrm{~h}$. Note the same distribution pattern as the EGFP-HCN4 constructs without extracellular HA-tag (Fig. 1A). (B), EGFP-HCN4-HA channels at the plasma membrane detected with an antibody targeting the extracellular HA-epitope. (C), Merge of (A) and (B) shows a distinct co-localization of HAtagged and EGFP-fused HCN4 channels at the plasma membrane, co-localization is indicated in white. (D), Magnification from (C). (E-F), Results from FACS analysis of wild-type EGFP-HCN4-HA and D553N mutant constructs. Transfected cells within the region of interest for EGFP (black bars) and cells labelled by anti-HA (grey bars) were counted and percentage of cells within these regions are displayed for (E) wildtype and (F) D553N. Both constructs were detected to the same extent at the plasma membrane.

by fluorescence microscopy (Fig. 2A-D). As previously shown [19], the introduced HAtag did not alter the trafficking of the HCN4 channels, since the HA-tagged channels had a similar cellular distribution pattern as EGFP-tagged wild-type channels (Figs. 1C, 2A). Channels localized at the plasma membrane were identified by analyzing the fluorescence of the secondary antibody (PE-Cy5) which was targeting the primary HA-antibody (Fig. 2B). As a prerequisite for the subsequent FACS analysis, detection of EGFP and HA of the EGFP-HCN4-HA construct revealed co-localization exclusively at the plasma membrane 
(Fig. 2C, D). Next, we determined the surface expression of EGFP-HCN4-HA and EGFP-D553NHA utilizing FACS (Fig. 2E, F). For FACS experiments two regions of interest were analyzed: (i) EGFP positive and (ii) PE-Cy5 positive cells. Within both chosen regions of interest, a minimum of 10,000 cells were counted. The distribution of the cells to the different regions of interest were analyzed to calculate the percentage of surface expression. For wild-type, $57.0 \pm 8.3 \%$ of the EGFP-HCN4-HA transfected cells were detected in the region of interest for PE-Cy5, indicating surface membrane localization of the channel (Fig. 2E). The EGFPD553N-HA construct showed a similar amount (50.0 $\pm 2.3 \%$ ) of PE-Cy5 labelled cells (Fig. $2 F$ ). Since the ratio of EGFP- to Cy5-positive cells was not significantly altered between wildtype and D553N channels, we conclude that there is no significant difference in surface expression. These data indicate that the disease causing mechanism must be different from a trafficking defect.

\section{Functional characterization of D553N mutant channels}

As we observed that D553N HCN4 channels reach the plasma membrane, we analyzed whether the mutants generate currents. Therefore, we injected Xenopus laevis oocytes with cRNA encoding for HCN4 or D553N. Oocytes injected with wild-type HCN4 cRNA gave rise to typical hyperpolarization activated inward $I_{\mathrm{f}}$-like currents (Fig. $3 \mathrm{~A}$, top). In contrast to previous reports $[10,17]$, D553N mutants generated a fair amount of current and current kinetics resembled those of wild-type HCN4 currents (Fig. 3A, bottom). However, D553N mutants conducted less current than wild-type channels (Fig. 3B). Current amplitudes were monitored for four subsequent days and the reduction in D553N currents as observed to a similar extent at any time point. Current amplitudes of both, wild-type and D553N channels, peaked the fifth day after cRNA injection. These data suggest that wild-type and D553N channels traffic similar, while D553N channels conduct less current.

HCN4 channels form functional heteromers with other HCN isoforms [20]. Therefore, we tested whether D553N subunits suppress the function of HCN subunits that are also present in the sino-atrial node tissue (Fig. 3C-E). In contrast to the co-transfection experiments described by Ueda et al. [10], co-expression of a controlled and fixed ratio of D553N with HCN4 in Xenopus oocytes did not show a dominant-negative reduction of wildtype HCN4 currents (Fig. 3C). Injection of $2.5 \mathrm{ng}$ of wild-type or D553N again revealed a strong reduction of current amplitudes for the mutant (Fig. 3C). Injection of half the amount of HCN4, mimicking a haploinsufficiency, produced currents with half of the magnitude (Fig. 3C). These data show that the co-expression experiments were performed in a linear range. However, co-expression of $1.25 \mathrm{ng}$ D553N with $1.25 \mathrm{ng}$ HCN4 did not result in a reduction of HCN4 currents, compared to the 1.25 ng of wild-type alone (Fig. 3C). These experiments demonstrate that D553N does not cause a dominant-negative suppression of HCN4 currents.

Next, we co-expressed D553N with other HCN isoforms and compared the current amplitudes to heteromeric channels containing wild-type HCN4 (Fig. 3D, E). Co-expression of $1.25 \mathrm{ng}$ of D553N with $1.25 \mathrm{ng}$ of HCN1 did not alter current amplitudes of heteromeric HCN1/HCN4 channels (Fig. 3D). In contrast, we found that co-expression of D553N with HCN2 strongly reduced current amplitudes of heteromeric HCN2/HCN4 channels (Fig. 3E). This dominant-negative reduction of heteromeric HCN2/HCN4 currents might contribute to the phenotype of patients with a D553N mutation.

Next, we analyzed the kinetics of wild-type and D553N currents. Activation kinetics were analyzed with a bi-exponential fit at different test potentials to determine the time constants of activation (Fig. 3F) and the corresponding relative contribution of the fast and slow components of activation (Fig. 3G). Furthermore, we analyzed the time constants of deactivation at $+20 \mathrm{mV}$ (Fig. $3 \mathrm{H}$ ). Analysis of current-voltage relationships (G/Vs) showed that wild-type channels open with a $V_{1 / 2}$ of $-92.2 \pm 0.6 \mathrm{mV}$ and $D 553 \mathrm{~N}$ with a $V_{1 / 2}$ of -93.9 $\pm 0.4 \mathrm{mV}$ (Fig. 3I). In addition, the Min- $\mathrm{P}_{\text {o }}$ for wild-type and D553N channels was analyzed (Fig. 3J). None of the determined parameters differed strongly between wild-type and the D553N mutant. Thus, we exclude changes in activation and deactivation kinetics of the D553N mutant as the primary disease causing mechanism. Since the current amplitude of 


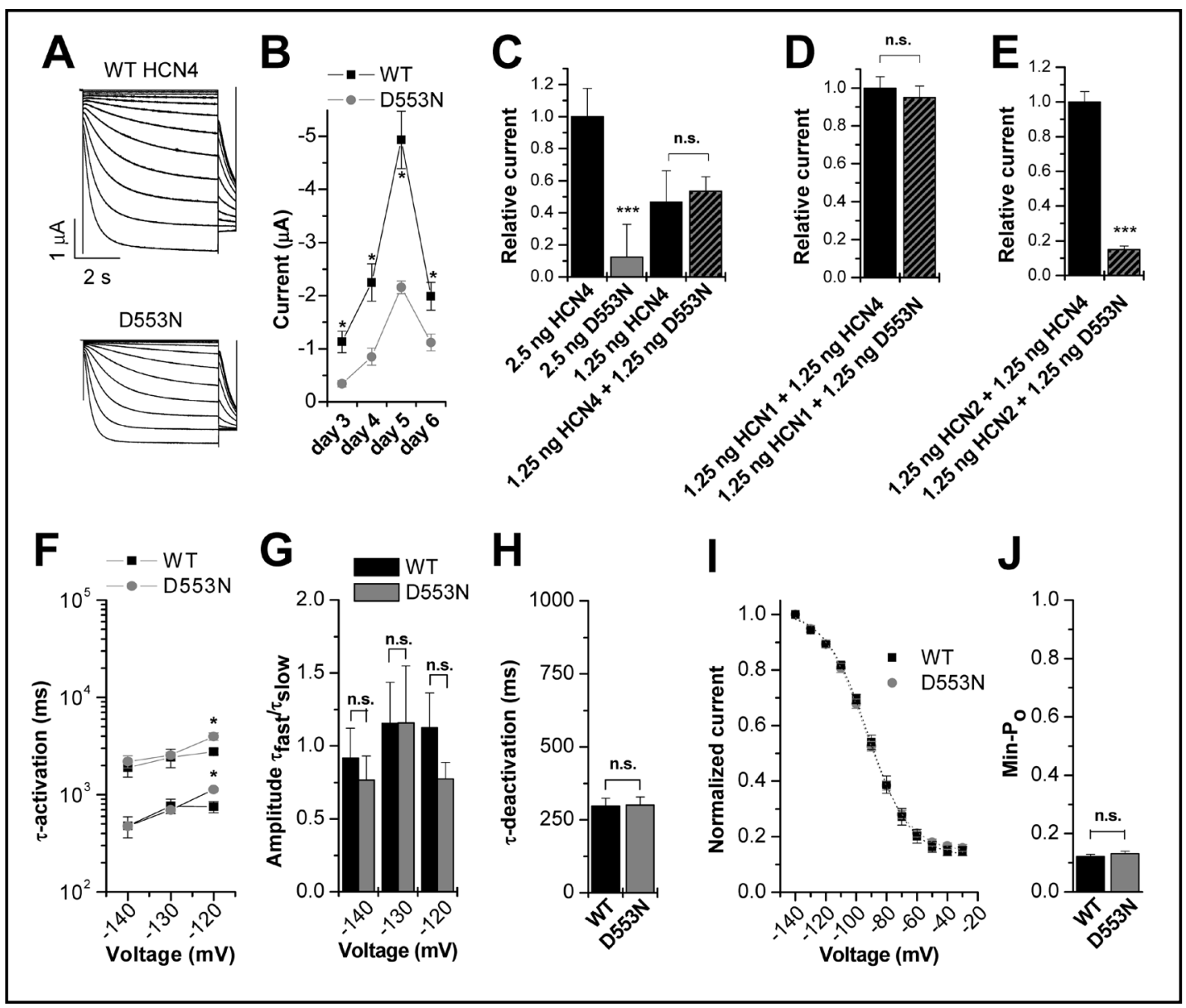

Fig. 3. Electrophysiological characterization of the D553N mutant in Xenopus oocytes. (A), Representative current traces of wild-type and D553N HCN4 expressed in Xenopus oocytes. Currents were recorded with voltage steps of $6 \mathrm{~s}$ duration to potentials between -30 and $-140 \mathrm{mV}$ in $-10 \mathrm{mV}$ increments, followed by an $800 \mathrm{~ms}$ step to $-130 \mathrm{mV}$, then back to $+20 \mathrm{mV}$. Holding potential was $-30 \mathrm{mV}$ with a sweep time interval of $12 \mathrm{~s}$. (B), Current amplitude measurements at $-130 \mathrm{mV}$ of wild-type (black) and D553N (grey) constructs injected in Xenopus oocytes, measured on four subsequent days ( $\mathrm{n}=13-41)$. (C), Co-expression experiments of D553N with HCN4 in Xenopus oocytes, analyzed at $-130 \mathrm{mV}$ at day 4 after injection $(\mathrm{n}=17-22)$. (D), Coexpression experiments of D553N with HCN1 and (E), HCN2 channels $(n=17-23)$. Current amplitudes were compared to the respective wild-type heteromeric channels. (F-J) Analysis of wild-type and D553N HCN4 current kinetics $(n=6-40)$. (F), Time constants of activation. $(G)$, Amplitude ratio for the fast and slow components of channel activation. $(\mathrm{H})$, Time constants of deactivation determined by a step of $6 \mathrm{~s}$ duration to $-130 \mathrm{mV}$ followed by a test pulse to $+20 \mathrm{mV}$. (I), G/V of wild-type and D553N HCN4 channels. (J), Min- $\mathrm{P}_{\mathrm{o}}$ of wild-type HCN4 $(\mathrm{n}=32)$ and D553N $(\mathrm{n}=40)$ channels.

HCN channels is regulated by tyrosine phosphorylation [21], we next assessed whether the reduced current amplitude of D553N channels is caused by altered phosphorylation.

D553N mutants have a regular modulation by tyrosine kinases

HCN4 channels may undergo a tyrosine phosphorylation at the residues Y531 and Y554 [22]. The C-terminal D553N exchange is located next to two tyrosine residues of HCN4: ${ }^{553} \mathrm{DYY}^{555}$. The corresponding sequence of HCN2 is ${ }^{475} \mathrm{D} p \mathrm{YY}^{477}$ and $\mathrm{Y} 476$ has been 


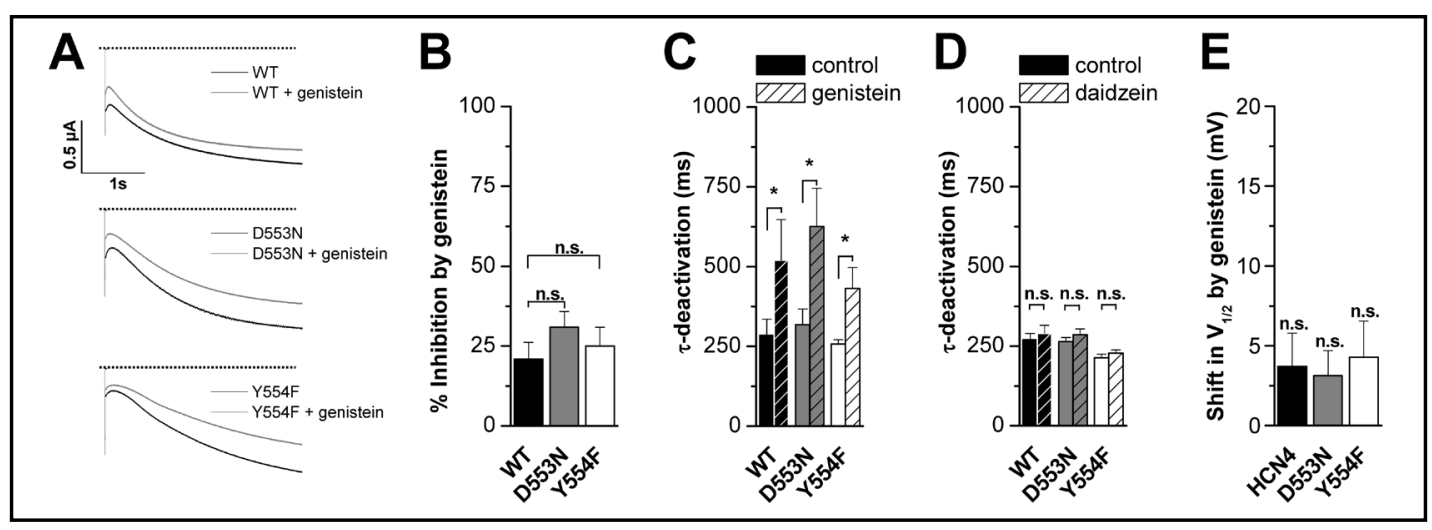

Fig. 4. Analyses of D553N channel modulation by tyrosine kinases. (A), Representative currents of wild-type, D553N and Y554F HCN4 channels recorded in Xenopus oocytes by a step to -130 mV, before (black) and after (grey) administration of $100 \mu$ M genistein. (B), Percentage of inhibition caused by genistein $(n=6-8)$. Time constants of deactivation in the absence and presence of genistein (C), or (D), daidzein $(n=6-8)$. (E), Shift of the $V_{1 / 2}$ of wild-type, D553N and Y554F by $100 \mu$ M genistein $(n=6-8)$.

described as a tyrosine phosphorylation site [6]. Therefore, we tested whether the reduced current amplitude of the D553N mutant is caused by a loss of phosphorylation at position Y554. We studied the Y554F mutant, as the mutation directly disrupts the putative target phosphorylation site. Accordingly, if defective tyrosine phosphorylation at Y554 causes the phenotype, Y554F should have the same phenotype as the D553N mutant. In addition, we analyzed the effect of the tyrosine kinase inhibitor genistein and the inactive isoform daidzein on wild-type, D553N and Y554F expressed in oocytes (Fig. 4A-E). Figure 4A shows representative current traces from wild-type, D553N and Y554F under control conditions (black) and after administration of $100 \mu \mathrm{M}$ genistein (grey). Currents of all constructs were significantly inhibited by genistein (Fig. 4B), but not by daidzein (data not shown). Genistein significantly slowed deactivation of all constructs (Fig. 4C), while daidzein had no effect (Fig. 4D). Genistein caused a non-significant shift of the $\mathrm{V}_{1 / 2}$ of wild-type HCN4, D553N and Y554F (Fig. 4E), similar as daidzein (data not shown). Summarizing, there is no significant difference in the tyrosine kinase modulation of wild-type, D553N, and Y554F HCN4 channels. Similar results were observed for the tyrosine kinase inhibitor PP2 and the inactive analog PP3 (data not shown). Therefore, we exclude a defective tyrosine phosphorylation at residue Y554 as the disease causing mechanism for the D553N mutation.

HCN channel activation is regulated by binding of cAMP to the CNBD. As the D553 residue is located in the C-linker, in close proximity to the CNBD, we next assessed whether D553N mutations might have an impaired regulation by cAMP.

\section{Altered modulation of the voltage dependence by cAMP cannot account for the major} current reduction of D553N

Modulation of HCN channels by cAMP is mediated by the cyclic nucleotide-binding domain (CNBD). Based on the crystal structure of the HCN2 and HCN4 C-termini [23], the residue D553 in the C-linker of HCN4 is in close proximity to the CNBD. As the C-linker is coupling cAMP binding of the CNBD to channel activation, we tested whether a reduced coupling after cAMP binding might be the disease causing mechanism. Therefore, we studied HCN4 and D553N mutant channels under basal and elevated cAMP levels, using IBMX in whole-cell patch-clamp recordings. In contrast to the initial report of Ueda et al., we found that D553N transfected COS-7 cells gave rise to relatively large macroscopic $I_{\mathrm{f}}$-like currents (Fig. 5A, D). D553N currents had an average amplitude of $406 \pm 92 \mathrm{pA}(\mathrm{n}=5)$ (Fig. 5D). While Ueda et al., observed an average conductance of about $3 \mathrm{pA} / \mathrm{pF}$ for D553N, we recorded currents with $15.6 \pm 5.7 \mathrm{pA} / \mathrm{pF}$ at $-120 \mathrm{mV}$. HCN4 and D553N current characteristics in 


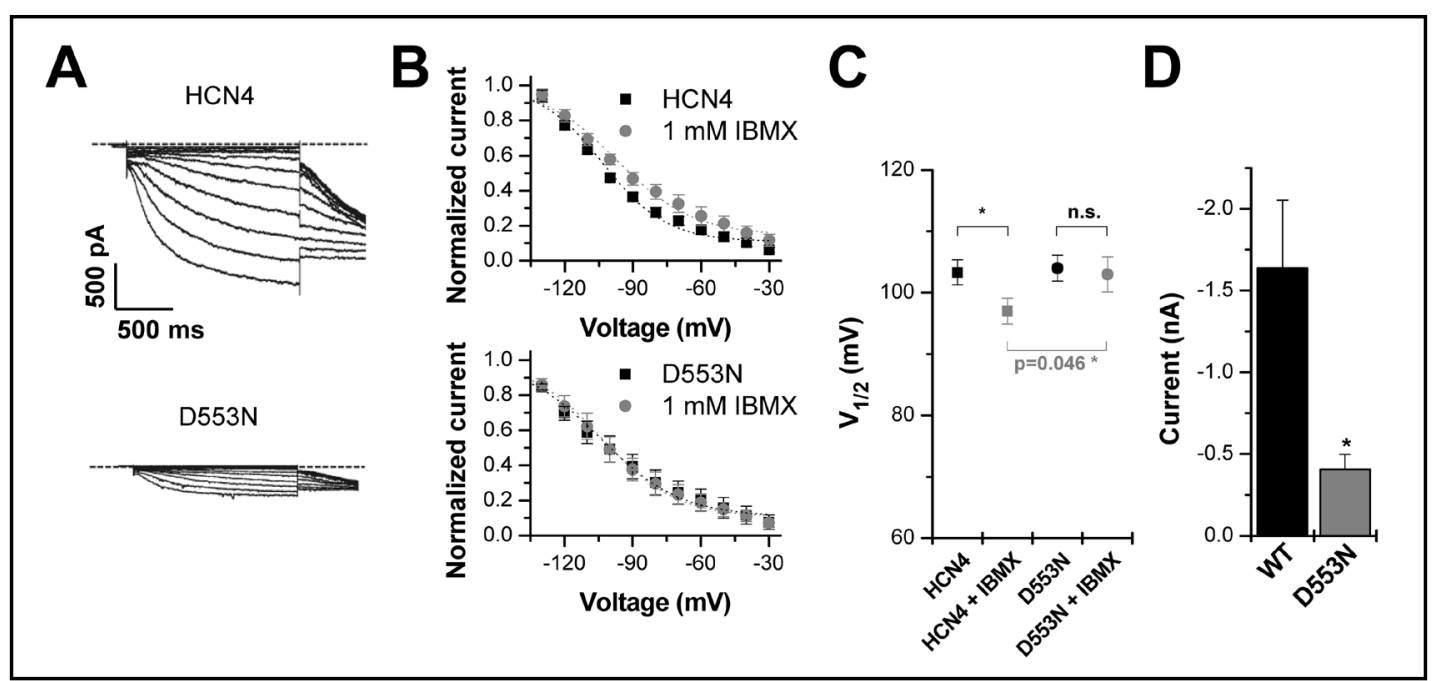

Fig. 5. Patch-clamp recordings of D553N in COS-7 cells reveal a reduced cAMP response. (A), Whole cell current traces of wild-type HCN4 (top) and D553N (bottom) mutant channels recorded by a protocol stepping from a holding potential of $-30 \mathrm{mV}$ to potentials ranging from $-30 \mathrm{mV}$ to $-140 \mathrm{mV}$ in $10 \mathrm{mV}$ increments for $2 \mathrm{~s}$ followed by a step to $-130 \mathrm{mV}$ for $300 \mathrm{~ms}$ with a $12 \mathrm{~s}$ sweep time interval. (B), G/Vs of wild-type (top) and D553N (bottom) before (black) and after (grey) administration of 1 mM IBMX are illustrated. (C), $\mathrm{V}_{1 / 2}$ values of wild-type channels and D553N mutants before and after administration of $1 \mathrm{mM}$ IBMX. (D), Bar graph showing the average current amplitudes for wild-type $(n=8)$ and D553N channels $(n=5)$, analyzed at $-130 \mathrm{mV}$.

transfected cells were similar to those recorded in Xenopus oocytes (Fig. 3) and no significant changes in activation and deactivation kinetics were observed (data not shown). Next, we measured current-voltage relationships before and after administration of $1 \mathrm{mM}$ IBMX (Fig. 5B, C). The $\mathrm{V}_{1 / 2}$ of wild-type HCN4 was shifted from $-103.3 \pm 2.0 \mathrm{mV}$ to $-97.0 \pm 2.1 \mathrm{mV}$ $(\mathrm{n}=4)$ (Fig. 5B,C). For D553N the voltage dependence was only shifted from $-104.0 \pm 2.1 \mathrm{mV}$ $(\mathrm{n}=4)$ to $-103.0 \pm 2.9 \mathrm{mV}(\mathrm{n}=4)$ (Fig. 5B, C). Thus, in the presence of $1 \mathrm{mM}$ IBMX, which generates high cAMP levels, there was a significant difference $(p=0.046)$ between the $V_{1 / 2}$ of wild-type HCN4 and D533N of about $6 \mathrm{mV}$ (Fig. 5C). More strikingly, similar as in our oocyte recordings (Fig. 3A, B), currents elicited by D553N mutants were strongly reduced compared to wild-type channels. The average current reduction by D553N, analyzed at -130 $\mathrm{mV}$, was $75.2 \%$ (Fig. 5D). Summarizing, the relatively minor change by an altered cAMP response of D553N cannot account for the strong current reduction which we have observed in our TEVC and patch-clamp recordings. As the current reduction of D553N is not caused by altered trafficking there must be a pronounced gating defect of D553N channels which is independent from the cAMP modulation.

\section{Mechanism of the loss-of-function on a single channel level?}

To probe the expected loss-of-function in channel gating, we performed single channel recordings in the cell-attached mode, using previously described recording conditions $[24,25]$. For these experiments, CHO cells were transiently transfected with wild-type or D553N channels. Unfortunately and despite major efforts, we were not able to record wildtype HCN4 single channels, even from cells which show clear macroscopic HCN4 whole cell currents. However, the literature on HCN4 single channel data is very conflicting [26] and only one group was so far able to record single HCN channels from transfected mammalian cell lines $[24,25]$. While the single channel amplitude of HCN channels recorded from this group was around $17 \mathrm{pS}[24,25]$, HCN channels in sino-atrial tissue recorded by DiFrancesco et al., were about $1 \mathrm{pS}[27,28]$. Thus, we and others failed to record the small typical HCN single channels described in native tissue. 
Summarizing, in contrast to the initial description of the D553N mutation, we found that the mutant does not cause a dominant-negative HCN4 trafficking defect. Our data support the role of the C-linker for HCN channel gating and that the loss-of-function in D553N mutants is presumably caused by channels with impaired function located at the surface membrane.

\section{Discussion}

Several HCN4 channel mutations have been associated with sino-atrial dysfunction $[3,11,12,29,30]$. All of these mutations cause a loss of channel function and most of the mutations are located in the C-terminus of HCN4 [3, 10-12]. The C-terminal HCN4 mutations S672R, 573X and 695X show a mild loss-of-function based on a defective cAMP modulation or an altered voltage dependence of activation $[3,11,12]$. In contrast, D553N was reported to result in a mis-trafficking of the channel [10], which acts in a dominant-negative manner leading to an almost complete loss of HCN4 channel function in heterozygous patients. The D553N mutation is located in the C-linker, connecting the S6 segment of the HCN4 channel with the CNBD. The C-linker is important for HCN and hERG channel gating [14-16, 23, 31]. In addition, the C-linker is thought to transduce cAMP binding to channel activation [15].

The observation that the D553N mutation in the C-linker of HCN4 is the only assumed trafficking-deficient and dominant-negative HCN4 mutation prompted us to further study the disease causing mechanism of this amino acid exchange [23]. In contrast to the initial report [10], we found that D553N channels reach the plasma membrane and do not act in a dominant-negative manner on wild-type HCN4. Our fluorescence microscopy data show that the cellular expression pattern and surface expression of the mutant was in fact not altered. The results from a subsequent FACS analysis support these findings. An open question is why D553N was initially reported to be trafficking defect and dominant-negative. Possible explanations are (a) Ueda et al. introduced the human D553N mutation into a rabbit HCN4 clone and not into the human orthologue and (b) the constructs were imaged and patchclamped with a C-terminal EGFP tag. Since the C-terminal region of ion channels mostly contain cellular trafficking motifs, the EGFP tag might have interfered with the regular HCN4 trafficking [19]. Unfortunately, in another study showing the non-functionality of D553N channels the position of the introduced DsRed tag was not provided [17].

To assess whether the D553N mutation destroys a putative tyrosine kinase site at position Y554, we analyzed the Y554F mutation and tested tyrosine kinase inhibitors on D553N channels. Our data indicate that D553N does not change the sensitivity of HCN4 channels to phosphorylation by tyrosine kinases. In contrast, Lin et al. recently showed that one can record an $I_{\mathrm{f}}$-like current in D553N transfected cells only after treatment with constitutively active isoforms of Src or Yes tyrosine kinases and interpreted these findings as a rescue of D553N channels [17]. However, HEK293 cells have been reported to express HCN2 and HCN3 channels endogenously [32]. Lin et al. did not test whether the kinases can induce $I_{\mathrm{f}}$-like currents in untransfected HEK293 cells. In addition, we were able to record D553N mutants also in the absence of tyrosine kinase activation. Our observation that D553N channels are not trafficking defect are also in line with the findings of Arinsburg et al. who showed that neither constitutively active, nor constitutively inactive isoforms of Src tyrosine kinases alter the current density of HCN4 channels [33].

Our data provide strong evidence that the disease causing mechanism of the D553N mutation is not mediated by a trafficking defect. This is supported by our patch-clamp and TEVC recordings which show that D553N channels are, unlike previously reported $[10,17]$, reaching the plasma membrane with a similar efficiency as wild-type channels. However, despite the normal current kinetics, the amplitudes of D553N currents were significantly reduced. We found that the mutation in the C-linker results in an altered sensitivity to raised cAMP levels. However, the $V_{1 / 2}$ values of D553N and wild-type channels under high cAMP levels ( $1 \mathrm{mM}$ IBMX) are only different by $6 \mathrm{mV}$. Accordingly, these minor effects cannot 


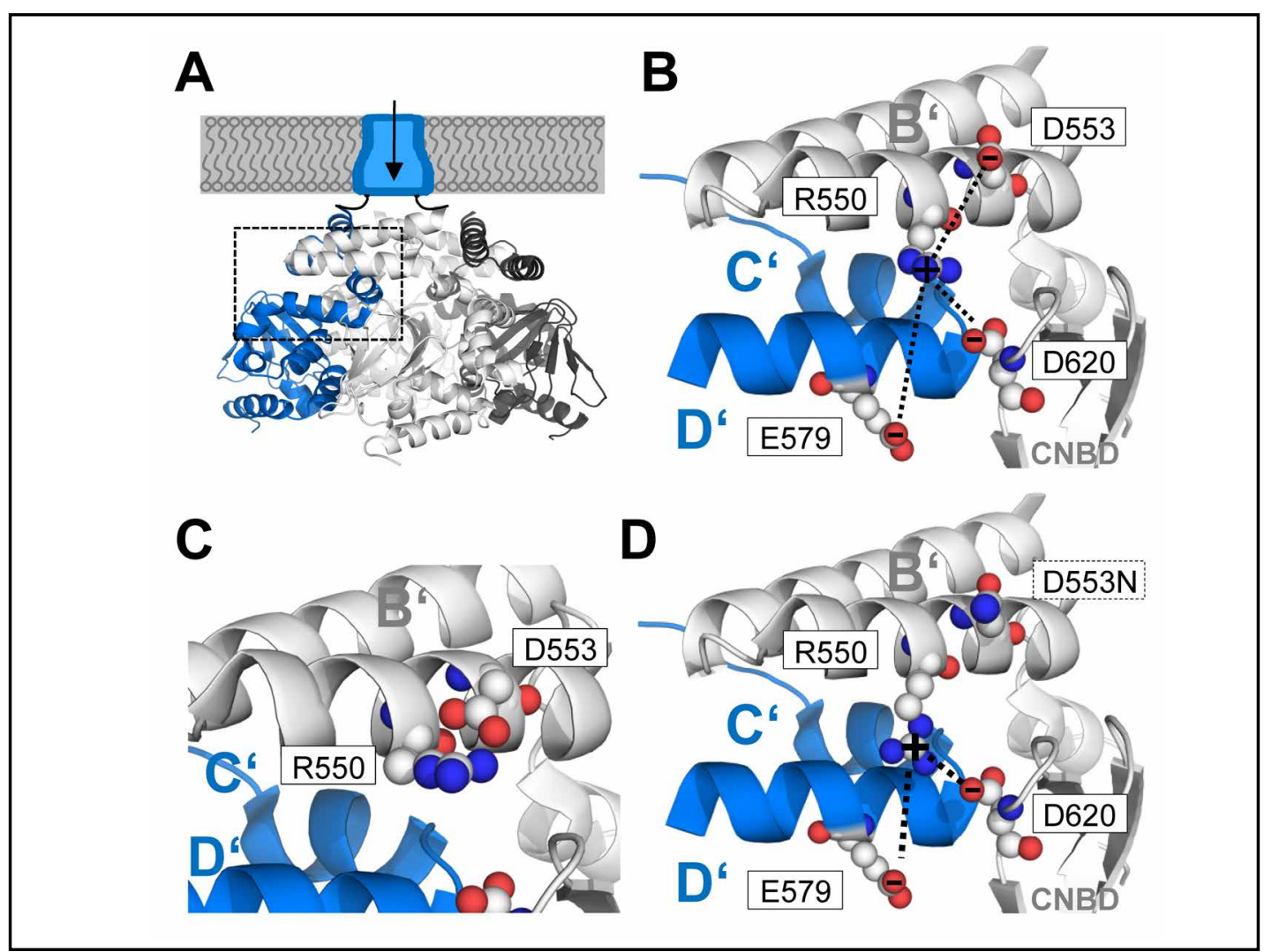

Fig. 6. A putative role of D553 in the interaction between the C-linker and CNBD of HCN4 channels. (A), Cartoon showing HCN4 (blue) and the crystal structure of the HCN4 C-terminus (PDB ID: 30TF). (B), Magnification of the area framed by the dotted box in (A) showing the intersubunit interaction of the B' strand (grey) to a D' strand (blue) of a neighbouring subunit, which reflects the "elbow" (B') on a "shoulder" ( $\mathrm{C}^{\prime}$ and $\mathrm{D}^{\prime}$ ) principle previously described [23]. A salt bridge between the R550 of the B' helix to E579 of the $\mathrm{D}^{\prime}$ helix and D620 of the $\beta$-sheet of the CNBD stabilizes the closed state. In HCN2 channels this triad of amino acids (E502, D542, K472) was described as a prerequisite for a proper gating of the channel $[15,16]$. The figures illustrate that D553 in HCN4 might interfere with this triad of amino acids. The salt bridge might be "weakened" by D553N located next to R550 (see dotted line for a putative electrostatic interaction). (C), Illustration that R550 and D553 can interact with each other as they are located only one helical turn apart. This electrostatic interaction of D553 with R550 might shield the R550 from interacting with the negative charges of the neighbouring subunit. (D), Accordingly, neutralization of the negative charge of D553 might stabilize the triad salt bridge and thus stabilize the closed state.

account for the strong current reduction observed for D553N. We therefore expect an additional gating defect, independent from cAMP modulation, to contribute to the disease causing mechanism which is mediated by a dysfunction of the C-linker.

In this context it is important to note, that the crystal structure of the HCN4 C-terminus [34] reveals that D553 is in close proximity to the CNBD of a neighbouring subunit (Fig. 6A, B). Disruption of an intersubunit salt bridge in this area leads to an impaired HCN and CNG channel gating [16]. This functionally relevant salt bridge is also present in HCN4 channels [34] and is located in close proximity to D553 (Fig. 6B). The residue R550 which forms the salt bridge with two negatively charged residues in the $\mathrm{D}^{\prime}$ helix and the CNBD (Fig. 6B) of a neighbouring subunit, is separated from D553 by only one helix turn on the B' helix (Fig. 6B). D553 and R550 are close enough for an electrostatic interaction (Fig. 6B, C). Thus, aspartate 553 might shield some of the positive charge of R550, as they can rotate and interact with each other (Fig. 6C). The neutralization of the negative charge at position 553 by the D553N exchange in turn might stabilize the intersubunit salt bridge described by Craven et al. [16]. 
Thus, it is conceivable that the D553N mutation stabilizes interaction of the C-linker with the CNBD of the HCN4 channel resulting in a more stable configuration that might impair channel gating (Fig. 6D). For example, this stabilization might restrict the movement of the C-linker, thereby possibly affecting the coupling from C-linker movement to the gate of the channel. In HCN2 channels disruption of this particular triad salt-bridge leads to a loss of cAMP response [16], which is in good agreement with our finding that the D553N mutation might also have an altered cAMP sensitivity.

Structurally, HCN2 and HCN4 both have the above mentioned intersubunit salt-bridge which might be also important for the function of heteromeric channels. In fact, our coexpression experiments revealed that D553N HCN4 channels suppress the function of heteromeric channels with HCN2. Why the D553N mutation only acts as a "poisonous pill" in complexes with HCN2 but not HCN4 remains an open question which we would like to address in future studies analyzing the mechanism of heteromerization of HCN channel subunits. However, from our recordings, we can already conclude that the patients with D553N might also suffer from a loss of heteromeric HCN2/HCN4 channel function.

We found that sino-atrial arrhythmias in patients carrying the D553N mutation are not caused by a dominant-negative trafficking defect. The mutation D553N is located in the C-linker of HCN4 channels. Structural changes in the C-linker can lead to impaired coupling of S6-movements to the pore, thereby changing the gating of hERG and HCN channels [14$16,31,35,36]$. Our data support the role of the C-linker for HCN channel gating and that the loss-of-function in D553N mutants is primarily caused by a gating defect.

\section{Acknowledgements}

We want to thank Bernd Fakler and Martin Biel for HCN4 expression constructs and William C. Claycomb for HL-1 cells. We would like to thank Kirsten Ramlow for excellent technical assistance. This work was supported by grants DE1482-2/1, DE1482-3/1 and DE1482-3/2 of the Deutsche Forschungsgemeinschaft (DFG) to N.D..

\section{Conflict of Interest}

None declared.

\section{References}

1 Sanguinetti MC, Tristani-Firouzi M: hERG potassium channels and cardiac arrhythmia. Nature 2006;440:463-469.

- Benson DW, Wang DW, Dyment M, Knilans TK, Fish FA, Strieper MJ, Rhodes TH, George AL Jr: Congenital sick sinus syndrome caused by recessive mutations in the cardiac sodium channel gene (SCN5A). J Clin Invest 2003;112:1019-1028.

-3 Schulze-Bahr E, Neu A, Friederich P, Kaupp UB, Breithardt G, Pongs O, Isbrandt D: Pacemaker channel dysfunction in a patient with sinus node disease. J Clin Invest 2003;111:1537-1545.

4 DiFrancesco D, Tortora P: Direct activation of cardiac pacemaker channels by intracellular cyclic AMP. Nature 1991;351:145-147.

- Z Zolles G, Klöcker N, Wenzel D, Weisser-Thomas J, Fleischmann BK, Röper J, Fakler B: Pacemaking by HCN channels requires interaction with phosphoinositides. Neuron 2006;52:1027-1036.

6 Zong X, Eckert C, Yuan H, Wahl-Schott C, Abicht H, Fang L, Li R, Mistrik P, Gerstner A, Much B, Baumann L, Michalakis S, Zeng R, Chen Z, Biel M: A novel mechanism of modulation of hyperpolarization-activated cyclic nucleotide-gated channels by Src kinase. J Biol Chem 2005;280:34224-34232. 


\section{Cellular Physiology Cell Physiol Biochem 2012;30:1227-1240 and Biochemistry

7 Baruscotti M, Bucchi A, Viscomi C, Mandelli G, Consalez G, Gnecchi-Rusconi T, Montano N, Casali KR, Micheloni S, Barbuti A, DiFrancesco D: Deep bradycardia and heart block caused by inducible cardiacspecific knockout of the pacemaker channel gene HCN4. Proc Natl Acad Sci USA 2011;108:1705-1710.

-8 Stieber J, Herrmann S, Feil S, Loster J, Feil R, Biel M, Hofmann F, Ludwig A: The hyperpolarization-activated channel HCN4 is required for the generation of pacemaker action potentials in the embryonic heart. Proc Natl Acad Sci U S A 2003;100:15235-15240.

-9 Ludwig A, Budde T, Stieber J, Moosmang S, Wahl C, Holthoff K, Langebartels A, Wotjak C, Munsch T, Zong X, Feil S, Feil R, Lancel M, Chien KR, Konnerth A, Pape HC, Biel M, Hofmann F: Absence epilepsy and sinus dysrhythmia in mice lacking the pacemaker channel HCN2. EMBO J 2003;22:216-224.

$>10$ Ueda K, Nakamura K, Hayashi T, Inagaki N, Takahashi M, Arimura T, Morita H, Higashiuesato Y, Hirano Y, Yasunami M, Takishita S, Yamashina A, Ohe T, Sunamori M, Hiraoka M, Kimura A: Functional characterization of a trafficking-defective HCN4 mutation, D553N, associated with cardiac arrhythmia. J Biol Chem 2004;279:27194-27198.

11 Milanesi R, Baruscotti M, Gnecchi-Ruscone T, DiFrancesco D: Familial sinus bradycardia associated with a mutation in the cardiac pacemaker channel. N Engl J Med 2006;354:151-157.

12 Schweizer PA, Duhme N, Thomas D, Becker R, Zehelein J, Draguhn A, Bruehl C, Katus HA, Koenen M: cAMP sensitivity of HCN pacemaker channels determines basal heart rate but is not critical for autonomic rate control. Circ Arrhythm Electrophysiol 2010;3:542-552.

13 Johnson JP Jr, Zagotta WN: The carboxyl-terminal region of cyclic nucleotide-modulated channels is a gating ring, not a permeation path. Proc Natl Acad Sci USA 2005;102:2742-2747.

14 Decher N, Chen J, Sanguinetti MC: Voltage-dependent gating of hyperpolarization-activated, cyclic nucleotide-gated pacemaker channels: molecular coupling between the S4-S5 and C-linkers. J Biol Chem 2004;279:13859-13865.

-15 Craven KB, Olivier NB, Zagotta WN: C-terminal movement during gating in cyclic nucleotide-modulated channels. J Biol Chem 2008;283:14728-14738.

-16 Craven KB, Zagotta WN: Salt bridges and gating in the COOH-terminal region of HCN2 and CNGA1 channels. J Gen Physiol 2004;124:663-677.

17 Lin YC, Huang J, Kan H, Frisbee JC, Yu HG: Rescue of a trafficking defective human pacemaker channel via a novel mechanism: roles of Src, Fyn, and Yes tyrosine kinases. J Biol Chem 2009;284:30433-30440.

18 Claycomb WC, Lanson NA Jr, Stallworth BS, Egeland DB, Delcarpio JB, Bahinski A, Izzo NJ Jr: HL-1 cells: a cardiac muscle cell line that contracts and retains phenotypic characteristics of the adult cardiomyocyte. Proc Natl Acad Sci U S A 1998;95:2979-2984.

19 Hardel N, Harmel N, Zolles G, Fakler B, Klöcker N: Recycling endosomes supply cardiac pacemaker channels for regulated surface expression. Cardiovasc Res 2008;79:52-60.

20 Much B, Wahl-Schott C, Zong X, Schneider A, Baumann L, Moosmang S, Ludwig A, Biel M: Role of subunit heteromerization and $\mathrm{N}$-linked glycosylation in the formation of functional hyperpolarization-activated cyclic nucleotide-gated channels. J Biol Chem 2003;278:43781-43786.

21 Huang J, Huang A, Zhang Q Lin YC, Yu HG: Novel mechanism for suppression of hyperpolarization-activated cyclic nucleotide-gated pacemaker channels by receptor-like tyrosine phosphatase-alpha. J Biol Chem 2008;283:29912-29919.

-22 Li CH, Zhang Q, Teng B, Mustafa SJ, Huang JY, Yu HG: Src tyrosine kinase alters gating of hyperpolarizationactivated HCN4 pacemaker channel through Tyr531. Am J Physiol Cell Physiol 2008;294:C355-362.

-23 Zagotta WN, Olivier NB, Black KD, Young EC, Olson R, Gouaux E: Structural basis for modulation and agonist specificity of HCN pacemaker channels. Nature 2003;425:200-205.

-24 Brandt MC, Endres-Becker J, Zagidullin N, Motloch LJ, Er F, Rottlaender D, Michels G, Herzig S, Hoppe UC: Effects of KCNE2 on HCN isoforms: distinct modulation of membrane expression and single channel properties. Am J Physiol Heart Circ Physiol 2009;297:H355-363.

25 Michels G, Er F, Khan I, Sudkamp M, Herzig S, Hoppe UC: Single-channel properties support a potential contribution of hyperpolarization-activated cyclic nucleotide-gated channels and $I_{\mathrm{f}}$ to cardiac arrhythmias. Circulation 2005;111:399-404.

26 DiFrancesco D: Letter regarding article by Michels et al, "Single-channel properties support a potential contribution of hyperpolarization-activated cyclic nucleotide-gated channels and If to cardiac arrhythmias". Circulation 2005;112:e72; author reply e72-73. 
27 DiFrancesco D: Characterization of single pacemaker channels in cardiac sino-atrial node cells. Nature 1986;324:470-473.

28 DiFrancesco D, Mangoni M: Modulation of single hyperpolarization-activated channels $I_{\mathrm{f}}$ by cAMP in the rabbit sino-atrial node. J Physiol 1994;474:473-482.

-29 Laish-Farkash A, Glikson M, Brass D, Marek-Yagel D, Pras E, Dascal N, Antzelevitch C, Nof E, Reznik H, Eldar M, Luria D: A novel mutation in the HCN4 gene causes symptomatic sinus bradycardia in moroccan jews. J Cardiovasc Electrophysiol 2010;21:1365-1372.

30 Nof E, Luria D, Brass D, Marek D, Lahat H, Reznik-Wolf H, Pras E, Dascal N, Eldar M, Glikson M: Point mutation in the HCN4 cardiac ion channel pore affecting synthesis, trafficking, and functional expression is associated with familial asymptomatic sinus bradycardia. Circulation 2007;116:463-470.

-31 Ferrer T, Rupp J, Piper DR, Tristani-Firouzi M: The S4-S5 linker directly couples voltage sensor movement to the activation gate in the human ether-a'-go-go-related gene (hERG) $\mathrm{K}^{+}$channel. J Biol Chem 2006;281:12858-12864.

-32 Varghese A, Tenbroek EM, Coles J Jr, Sigg DC: Endogenous channels in HEK cells and potential roles in HCN ionic current measurements. Prog Biophys Mol Biol 2006;90:26-37.

33 Arinsburg SS, Cohen IS, Yu HG: Constitutively active Src tyrosine kinase changes gating of HCN4 channels through direct binding to the channel proteins. J Cardiovasc Pharmacol 2006; 47:578-586.

34 Xu X, Vysotskaya ZV, Liu Q, Zhou L: Structural basis for the cAMP-dependent gating in the human HCN4 channel. J Biol Chem 2010;285:37082-37091.

-35 Chen J, Mitcheson JS, Tristani-Firouzi M, Lin M, Sanguinetti MC: The S4-S5 linker couples voltage sensing and activation of pacemaker channels. Proc Natl Acad Sci U S A 2001;98:11277-11282.

36 Tristani-Firouzi M, Chen J, Sanguinetti MC: Interactions between S4-S5 linker and S6 transmembrane $\checkmark$ domain modulate gating of HERG K+ channels. J Biol Chem 2002;277:18994-19000. 